\title{
Goal-directed haemodynamic therapy (GDHT) in surgical patients: systematic review and meta-analysis of the impact of GDHT on post-operative pulmonary complications
}

Ahilanandan Dushianthan ${ }^{1,2,3^{*}}$, Martin Knight ${ }^{1} \mathbb{D}$, Peter Russell ${ }^{1}$ and Michael PW Grocott ${ }^{1,2,3}$

\begin{abstract}
Background: Perioperative goal-directed haemodynamic therapy (GDHT), defined as the administration of fluids with or without inotropes or vasoactive agents against explicit measured goals to augment blood flow, has been evaluated in many randomised controlled trials (RCTs) over the past four decades. Reported post-operative pulmonary complications commonly include chest infection or pneumonia, atelectasis, acute respiratory distress syndrome or acute lung injury, aspiration pneumonitis, pulmonary embolism, and pulmonary oedema. Despite the substantial clinical literature in this area, it remains unclear whether their incidence is reduced by GDHT. This systematic review aims to determine the effect of GDHT on the respiratory outcomes listed above, in surgical patients.

Methods: We searched the Cochrane Central Register for Controlled Trials (CENTRAL), MEDLINE, EMBASE, and clinical trial registries up until January 2020. We included all RCTs reporting pulmonary outcomes. The primary outcome was post-operative pulmonary complications and secondary outcomes were specific pulmonary complications and intra-operative fluid input. Data synthesis was performed on Review Manager and heterogeneity was assessed using $P$ statistics.

(Continued on next page)
\end{abstract}

\footnotetext{
* Correspondence: a.dushianthan@soton.ac.uk

${ }^{1}$ General Intensive Care Unit, University Hospital Southampton NHS

Foundation Trust, Southampton SO16 6YD, UK

${ }^{2}$ Anaesthesia Perioperative and Critical Care Research Group, Southampton

NIHR Biomedical Research Centre, University Hospital Southampton/

University of Southampton, Southampton, UK

Full list of author information is available at the end of the article
}

(c) The Author(s). 2020 Open Access This article is licensed under a Creative Commons Attribution 4.0 International License, which permits use, sharing, adaptation, distribution and reproduction in any medium or format, as long as you give appropriate credit to the original author(s) and the source, provide a link to the Creative Commons licence, and indicate if changes were made. The images or other third party material in this article are included in the article's Creative Commons licence, unless indicated otherwise in a credit line to the material. If material is not included in the article's Creative Commons licence and your intended use is not permitted by statutory regulation or exceeds the permitted use, you will need to obtain permission directly from the copyright holder. To view a copy of this licence, visit http://creativecommons.org/licenses/by/4.0/. The Creative Commons Public Domain Dedication waiver (http://creativecommons.org/publicdomain/zero/1.0/) applies to the data made available in this article, unless otherwise stated in a credit line to the data. 
(Continued from previous page)

Results: We identified 66 studies with 9548 participants reporting pulmonary complications. GDHT resulted in a significant reduction in total pulmonary complications (OR 0.74, 95\% Cl 0.59 to 0.92). The incidence of pulmonary infections, reported in 45 studies with 6969 participants, was significantly lower in the GDHT group (OR 0.72, Cl 0.60 to 0.86). Pulmonary oedema was recorded in 23 studies with 3205 participants and was less common in the GDHT group (OR 0.47, Cl 0.30 to 0.73). There were no differences in the incidences of pulmonary embolism or acute respiratory distress syndrome. Sub-group analyses demonstrated: (i) benefit from GDHT in general/abdominal/mixed and cardiothoracic surgery but not in orthopaedic or vascular surgery; and (ii) benefit from fluids with inotropes and/or vasopressors in combination but not from fluids alone. Overall, the GDHT group received more colloid $(+280 \mathrm{ml})$ and less crystalloid $(-375 \mathrm{ml})$ solutions than the control group. Due to clinical and statistical heterogeneity, we downgraded this evidence to moderate.

Conclusions: This systematic review and meta-analysis suggests that the use of GDHT using fluids with inotropes and/or vasopressors, but not fluids alone, reduces the development of post-operative pulmonary infections and pulmonary oedema in general, abdominal and cardiothoracic surgical patients. This evidence was graded as moderate.

PROSPERO registry reference: CRD42020170361

Keywords: Goal-directed, Fluid therapy, Surgery

\section{Introduction}

Albeit uncommon, major surgery can be associated with significant pulmonary complications, increasing morbidity and mortality with a consequent greater burden on resources.(Pearse et al., 2012; Canet et al., 2010; Eappen et al., 2013) Goal-directed haemodynamic therapy (GDHT) describes the application of haemodynamic management to target-specific goals with the aim of improving global blood flow, and hence oxygen delivery, to the tissues. Previous systematic reviews support the notion that GDHT reduces post-operative complications following major surgery.(Hamilton et al., 2011) The role of GDHT in improving pulmonary outcomes following major surgery is uncertain.

The reported incidence of post-operative pulmonary complications varies depending on several factors including the definitions utilised, type of surgery, and patient-related variables including age, body mass index (BMI), functional reserve, smoking status, pre-existing lung diseases and the presence of obstructive sleep apnoea. The commonly reported pulmonary complications are atelectasis, respiratory tract infection/pneumonia, aspiration pneumonitis, acute respiratory distress syndrome (ARDS)/acute lung injury (ALI), pulmonary embolism and pulmonary oedema. Strategies to prevent or minimise post-operative pulmonary morbidity include pre-operative optimisation of pre-existing lung conditions, smoking cessation, pre-habilitation exercise programmes and intra-operative anaesthetic techniques such as increased use of neuraxial techniques, reduced use of long-acting neuromuscular blockade and lungprotective ventilation.(Ruscic et al., 2017)

In a Cochrane systematic review, the use of goaldirected fluid therapy was associated with a significant reduction in the incidence of respiratory failure.(Grocott et al., 2012) However, the effect of goal-directed fluid therapy on overall respiratory morbidity following surgery is unknown. Since the publication of the Cochrane review in 2012, many more randomised controlled trials have been published investigating the clinical impact of goal-directed haemodynamic therapy on post-operative outcomes, including respiratory complications.(Chong et al., 2018) The primary objective of this systematic review and meta-analysis is to investigate the effect of GDHT, defined by specific goals to augment blood flow, on reported post-operative pulmonary complications in patients undergoing surgery.

\section{Methods}

We performed a systematic review and meta-analysis of all relevant published randomised controlled trials. We adhered to the Cochrane and PRISMA statement in reporting this systematic review and meta-analysis. We conducted a systematic search on Ovid MEDLINE (January 03, 2020), EMBASE (week 52 2019) and the Cochrane Central Register of Controlled Trials (January 03, 2020). The search strategies used are available in the supplementary material. The protocol for this study is registered and accessible on the PROSPERO (International Prospective Register of Systematic Reviews) Registry (CRD42020170361).

\section{Inclusion and exclusion criteria}

We included all relevant randomised controlled trials with or without blinding and published in the English language. The participants were adults (age $>16$ years) undergoing any elective or emergency surgery (abdominal, vascular, orthopaedic, cardiac, thoracic and mixed groups). Studies of non-surgical patients with trauma or sepsis were excluded. Peri-operative goal-directed haemodynamic therapy was defined as administration of fluids, with or without inotropes or vasoactive agents, against explicit measured goals to augment blood flow and initiated in the pre-operative $(\leq 24 \mathrm{~h})$, intra-operative 
or post-operative period $(\leq 6 \mathrm{~h})$. We included all invasive and non-invasive monitoring devices used with a predefined algorithm to augment flow towards specific haemodynamic targets including the following measured or derived variables: cardiac output $(\mathrm{CO})$, stroke volume $(\mathrm{SV})$, oxygen delivery $\left(\mathrm{DO}_{2}\right)$, oxygen consumption $\left(\mathrm{VO}_{2}\right)$, mixed venous oxygen saturation and oxygen extraction ratio $\left(\mathrm{O}_{2} \mathrm{ER}\right)$. We grouped haemodynamic monitoring techniques into 4 categories: pulmonary artery catheter, oesophageal Doppler, minimally invasive and non-invasive. We defined minimally invasive techniques as using devices that require insertion of an arterial line and/or central venous access (but did not require a pulmonary artery catheter) and used arterial pulse contour analysis, transpulmonary thermo- or lithium dilution to measure dynamic cardiac variables (calibrated or uncalibrated). More recently, studies have evaluated hemodynamic monitoring techniques with the use of bioimpedance/bioreactance and non-invasive pulse contour analysis. These devices were defined as noninvasive cardiac output monitoring. Studies based on a dynamic assessment of plasma volume and fluid responsiveness such as systolic pressure variation (SPV), pulse pressure variation (PPV) or plethysmography variability index (PVI) were excluded as they do not provide a measure of flow.

\section{Data collection}

Two review authors (PR and MK) independently screened titles and abstracts from the search results to identify all potential studies. The studies were selected according to the inclusion and exclusion criteria and a third independent author (AD) further assessed studies against the eligibility criteria and resolved any disagreements by discussion. From the eligible studies, relevant data such as methods (study design, setting, number of centres), type of surgery and participants, interventions (goal-directed haemodynamic therapy algorithm and devices) and outcomes were extracted. Where data was missing, incomplete or grouped with other outcomes, we attempted to contact authors to obtain additional details if the study was within the 10 -year period.

\section{Types of outcome measures}

We included all studies that reported post-operative pulmonary complications. Our primary outcome was to assess the impact of goal-directed haemodynamic therapy on the incidence of post-operative pulmonary complications. This was presented as the total number of pulmonary events. We assessed individual events as secondary outcomes, as reported by the study authors. We used author-defined definitions for pneumonia, ARDS or ALI, pulmonary oedema, and pulmonary embolism. As it is often difficult clinically to distinguish pulmonary oedema associated with lung injury from fluid overload/hydrostatic or cardiogenic pulmonary oedema, we included this outcome as a pulmonary complication unless otherwise stated as cardiogenic in origin. We also performed subgroup analyses for the type of surgery, the haemodynamic monitoring device used, and the GDHT intervention delivered (fluids, or fluids and inotropes/vasopressors). We assessed intra-operative fluid administration, separating the use of crystalloids and colloids during the study period when reported.

\section{Assessment of risk of bias}

We used the Cochrane risk of bias tool to assess the quality of included studies.(Higgins \& Green, 2011) The risk of bias assessment consisted of the quality of randomisation and allocation concealment, blinding of participants and outcome assessors, selective or incomplete outcome reporting and any other potential sources of bias such as deviation from protocol, issues with the conduct of the study or influence from industry funding. We graded these components as "low risk", "high risk" or "unclear risk".

\section{Statistical analysis}

Quantitative synthesis was performed on the RevMan 5.3 software (Review Manager 2014). To assess our dichotomous data, we used Odds Ratios (OR), using the Mantel-Haenszel method with random-effects and fixedeffects models with 95\% confidence intervals (CI). Where possible, all our analysis was intention-to-treat (ITT). We assessed statistical heterogeneity by using $I^{2}$ statistics.(Higgins \& Green, 2011) Statistical heterogeneity was assumed when this value was $>40 \%$ and we performed random-effects models for these analyses. When $I^{2}$ was $<40 \%$, we used a fixed-effect model. Clinical heterogeneity was explored and addressed where possible by subgroup analysis. Consequently, we conducted subgroup analyses for the categories of surgery and the types of device utilised for the measurement of the specific haemodynamic goals. We encountered unit analysis issues, where some studies reported the number of patients with pulmonary complications, whilst others reported the total number of events within a population. Although we have attempted to analyse these separately, we did not proceed as there were only three studies that reported the number of patients with pulmonary complications. Data was transformed when no additional information was available to generate standard deviation and mean values from the median data presented (interquartile range, range, 95\% confidence intervals, and 25th and 75th percentiles), as guided by the Cochrane Collaboration.(Higgins \& Green, 2011) The quality of evidence was assessed by the Grading of Recommendations 
Assessment, Development and Evaluation (GRADE) system for the key outcomes reported.(Guyatt et al., 2008)

\section{Results}

Search results and study characteristics

The electronic search yielded a total of 18341 citations from electronic searches and additional records identified from other sources. After the removal of duplicates and screening by title and abstracts, we retrieved 150 studies for detailed review. We further excluded abstract publications $(N=30)$, dynamic studies which track pulse pressure or systolic pressure variations with no surrogate indices for global blood flow $(N=13)$ and studies that used additional goal-directed measures in the control group or compared different fluid solutions in the GDHT setting $(N=11)$. Ninety-six randomised controlled trials fulfilled our inclusion criteria and of those 67 studies reported post-operative pulmonary complications. One study reported pooled cardiac and respiratory complications and as a result, this was not included for quantitative data synthesis.(Srinivasa et al., 2013) We identified 6 ongoing trials (Table 1).

We also screened references from all eligible publications and other systematic reviews for additional publications. The PRISMA flow chart for the study search flow diagram is presented in Fig. 1.

A total of 66 randomised controlled trials with 9548 participants reported respiratory complications as part of their outcome reporting. A summary table of study characteristics is presented in Table 2, and an additional detailed table is available in the Supplementary material (Table A). The type of surgery performed was general/ mixed group or abdominal (44 studies)(Ackland et al., 2015; Bahlmann et al., 2019; Benes et al., 2010; Boyd et al., 1993; Brandstrup et al., 2012; Calvo-Vecino et al., 2018; Colantonio et al., 2015; Correa-Gallego et al., 2015; Donati et al., 2007; El Sharkawy et al., 2013; Gan et al., 2002; Gerent et al., 2018; Gomez-Izquierdo et al., 2017; Jammer et al., 2010; Jammer et al., 2015; Joosten et al., 2019; Kim et al., 2018; Kumar et al., 2016; Lobo et al., 2000; Luo et al., 2017; Mayer et al., 2010; McKenny et al., 2013; Mikor et al., 2015; Pearse et al., 2005; Pearse et al., 2014; Pestana et al., 2014; Phan et al., 2014; Reisinger et al., 2017; Salzwedel et al., 2013; Sandham et al., 2003; Scheeren et al., 2013; Schmid et al., 2016; Senagore et al., 2009; Shoemaker et al., 1988; Stens et al., 2017; Ueno et al., 1998; Wakeling et al., 2005; Weinberg et al., 2017; Weinberg et al., 2019; Wilson et al., 1999; Wu et al., 2017; Yin et al., 2018; Zakhaleva et al., 2013; Zeng et al., 2014), orthopaedic (9 studies)(Bartha et al., 2013; Cecconi et al., 2011; Davies et al., 2019; Han et al., 2016; Kaufmann et al., 2018; Moppett et al., 2015; Peng et al., 2014; Sinclair et al., 1997; Venn et al., 2002), cardiothoracic (7 studies) (Goepfert et al.,
Table 1 Ongoing studies identified from trial registries

\begin{tabular}{|c|c|c|}
\hline Title & Registry Number & $\begin{array}{l}\text { Expected } \\
\text { completion }\end{array}$ \\
\hline $\begin{array}{l}\text { A clinical trial of blood flow } \\
\text { optimisation for patients who have } \\
\text { emergency bowel surgery (FLOELA) }\end{array}$ & ISRCTN14729158 & $\begin{array}{l}\text { January } \\
2022\end{array}$ \\
\hline $\begin{array}{l}\text { Optimisation of perioperative } \\
\text { cardiovascular management to } \\
\text { improve surgical outcome II } \\
\text { (OPTIMISE II) }\end{array}$ & ISRCTN39653756 & $\begin{array}{l}\text { September } \\
2021\end{array}$ \\
\hline $\begin{array}{l}\text { Effect of SW-guided fluid therapy on } \\
\text { outcomes after major abdominal } \\
\text { surgery }\end{array}$ & NCT03940144 & $\begin{array}{l}\text { August } \\
2020\end{array}$ \\
\hline $\begin{array}{l}\text { Management of intraoperative fluids } \\
\text { in ambulatory surgery (MIFAS) }\end{array}$ & NCT03193320 & $\begin{array}{l}\text { December } \\
2020\end{array}$ \\
\hline $\begin{array}{l}\text { Effects of goal-directed fluid therapy } \\
\text { on postoperative outcomes in pa- } \\
\text { tients undergoing laparoscopic colo- } \\
\text { rectal surgery in different levels of } \\
\text { systemic vascular resistance after gen- } \\
\text { eral anaesthesia }\end{array}$ & ChiCTR1900022775 & Ongoing \\
\hline $\begin{array}{l}\text { The Application of Goal-directed fluid } \\
\text { therapy in the Fast-track Anaesthesia } \\
\text { for Gastrectomy }\end{array}$ & $\begin{array}{l}\text { ChiCTR-INR- } \\
17010636\end{array}$ & Ongoing \\
\hline
\end{tabular}

2013; Kaufmann et al., 2017; McKendry et al., 2004; Mythen \& Webb, 1995; Osawa et al., 2016; Xu et al., 2017; Zhang et al., 2013), and vascular (6 studies)(Bender et al., 1997; Bisgaard et al., 2013b; Boyd et al., 1993; Funk et al., 2015; Valentine et al., 1998; Van der Linden et al., 2010). Five studies were solely conducted on orthopaedic emergency patients(Bartha et al., 2013; Davies et al., 2019; Moppett et al., 2015; Sinclair et al., 1997; Venn et al., 2002), six additional studies included emergency surgical patients(Boyd et al., 1993; McKendry et al., 2004; Pearse et al., 2005; Pearse et al., 2014; Sandham et al., 2003; Shoemaker et al., 1988) and the rest were primarily conducted on elective surgical patients. The total sample size was $<100$ participants for 33 studies(Bahlmann et al., 2019; Bisgaard et al., 2013a; Bisgaard et al., 2013b; Cecconi et al., 2011; Colantonio et al., 2015; El Sharkawy et al., 2013; Funk et al., 2015; Han et al., 2016; Jammer et al., 2015; Joosten et al., 2019; Kaufmann et al., 2018; Kim et al., 2018; Kumar et al., 2016; Lobo et al., 2000; Mayer et al., 2010; Mikor et al., 2015; Mythen \& Webb, 1995; Peng et al., 2014; Reisinger et al., 2017; Scheeren et al., 2013; Senagore et al., 2009; Shoemaker et al., 1988; Sinclair et al., 1997; Ueno et al., 1998; Van der Linden et al., 2010; Venn et al., 2002; Weinberg et al., 2017; Weinberg et al., 2019; Wu et al., 2017; Yin et al., 2018; Zakhaleva et al., 2013; Zeng et al., 2014; Zhang et al., 2013) (50\%) and the majority (77\%) were conducted in a single centre setting(Bartha et al., 2013; Bender et al., 1997; Benes et al., 2010; Bisgaard et al., 2013a; Bisgaard et al., 2013b; Boyd et al., 1993; Cecconi et al., 2011; Colantonio et al., 2015; CorreaGallego et al., 2015; El Sharkawy et al., 2013; Funk et al., 


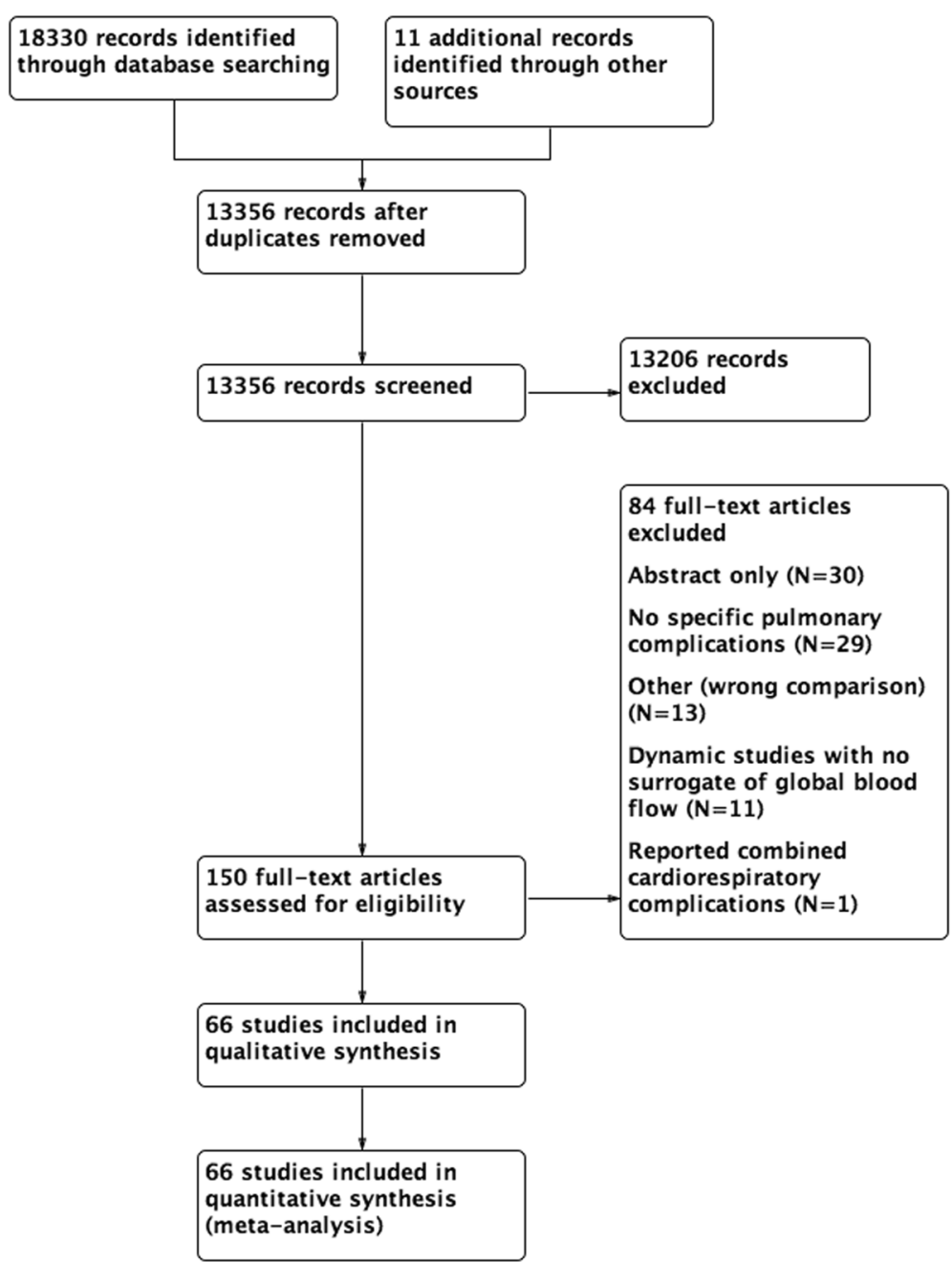

Fig. 1 PRISMA study search flow diagram

2015; Gan et al., 2002; Gerent et al., 2018; Goepfert et al., 2013; Gomez-Izquierdo et al., 2017; Han et al., 2016; Joosten et al., 2019; Kaufmann et al., 2017; Kaufmann et al., 2018; Kim et al., 2018; Kumar et al., 2016; Lobo et al., 2000; Luo et al., 2017; Mayer et al., 2010; McKendry et al., 2004; McKenny et al., 2013; Mikor et al., 2015; Moppett et al., 2015; Mythen \& Webb, 1995; Osawa et al., 2016; Pearse et al., 2005; Peng et al., 2014; Phan et al., 2014; Reisinger et al., 2017; Salzwedel et al., 2013; Schmid et al., 2016; Senagore et al., 2009; Shoemaker et al., 1988; Sinclair et al., 1997; Ueno et al., 1998; Valentine et al., 1998; Van der Linden et al., 2010; Venn et al., 2002; Wakeling et al., 2005; Wilson et al., 1999; Wu et al., 2017; Xu et al., 2017; Yin et al., 2018; Zakhaleva et al., 2013; Zeng et al., 2014; Zhang et al., 2013). The monitoring device for the majority of the studies was minimally invasive (33 studies)(Ackland et al., 2015; Bahlmann et al., 2019; Bartha et al., 2013; Benes et al., 2010; Bisgaard et al., 2013a; Bisgaard et al., 2013b; Cecconi et al., 2011; Colantonio et al., 2015; Correa-Gallego et al., 2015; Funk et al., 2015; Gerent et al., 2018; Goepfert et al., 2013; Han et al., 2016; Jammer et al., 2015; Kim et al., 2018; Kumar et al., 2016; Luo et al., 2017; Mayer et al., 2010; Moppett et al., 2015; Osawa et al., 2016; Pearse et al., 2005; Pearse et al., 2014; Peng et al., 2014; Salzwedel et al., 2013; Scheeren et al., 2013; Schmid et al., 2016; Van der Linden et al., 2010; Weinberg et al., 2017; Weinberg et al., 2019; Wu et al., 2017; Xu et al., 2017; Zeng et al., 2014; Zhang et al., 2013), followed by oesophageal Doppler (17 studies)(Brandstrup et al., 2012; Calvo-Vecino et al., 2018; El Sharkawy et al., 2013; Gan et al., 2002; GomezIzquierdo et al., 2017; Kaufmann et al., 2017; Kaufmann et al., 2018; McKendry et al., 2004; McKenny et al., 2013; Mythen \& Webb, 1995; Phan et al., 2014; Reisinger et al., 2017; Senagore et al., 2009; Sinclair et al., 1997; 
Table 2 Summary characteristics of included studies.

\begin{tabular}{|c|c|c|c|c|c|c|}
\hline Author/year & $\begin{array}{l}\text { No. of } \\
\text { patients }\end{array}$ & $\begin{array}{l}\text { Single/ } \\
\text { multicenter }\end{array}$ & $\begin{array}{l}\text { Type of } \\
\text { surgery }\end{array}$ & Timing & $\begin{array}{l}\text { Fluids/fluids \& inotropes (I) or } \\
\text { vasopressors (V) }\end{array}$ & Goals \\
\hline Ackland, 2015 & 204 & Multi & Elective & Post-operative & Fluids \& IN & $\begin{array}{l}\text { SV, CO, } \\
\text { DO2I }\end{array}$ \\
\hline Bahlmann, 2019 & 59 & Multi & Elective & Intra/post-operative & Fluids \& IN & SV, Cl, MAP \\
\hline Bartha, 2013 & 149 & Single & Emergency & Pre/intra-operative & Fluids \& IN & $\mathrm{SV}, \mathrm{DO}_{2} \mathrm{I}$ \\
\hline Bender 1997 & 104 & Single & Elective & $\begin{array}{l}\text { Pre/intra/post- } \\
\text { operative }\end{array}$ & Fluids \& IN & $\begin{array}{l}\text { SVR, } \mathrm{Cl}, \\
\text { PAOP }\end{array}$ \\
\hline Benes, 2010 & 120 & Single & Elective & Intra-operative & Fluids \& IN & $\mathrm{SW}, \mathrm{Cl}$ \\
\hline Bisgaard, 2013a, b & 70 & Single & Elective & Intra/post-operative & Fluids \& IN & $\mathrm{SVI}, \mathrm{DO}_{2} \mathrm{I}$ \\
\hline Bisgaard, 2013a, b & 40 & Single & Elective & Intra/post-operative & Fluids \& IN & $\mathrm{SVI}, \mathrm{DO}_{2} \mathrm{I}$ \\
\hline Boyd, 1993 & 107 & Single & Mixed & $\begin{array}{l}\text { Pre/intra/post- } \\
\text { operative }\end{array}$ & Fluids \& IN & $\mathrm{DO}_{2} \mathrm{I}$ \\
\hline Brandstrup, 2012 & 151 & Multi & Elective & Intra-operative & Fluids & SV \\
\hline Calvo-Vecino, 2018 & 450 & Multi & Elective & Intra-operative & Fluids \& IN & $\mathrm{SV}, \mathrm{Cl}$ \\
\hline Cecconi, 2011 & 40 & Single & Elective & Intra/post-operative & Fluids \& IN & $\mathrm{SV}, \mathrm{DO}_{2} \mathrm{I}$ \\
\hline Colantonio, 2015 & 86 & Single & Elective & Intra-operative & Fluids \& IN & $\mathrm{SVI}, \mathrm{Cl}$ \\
\hline $\begin{array}{l}\text { Correa-Gallego, } \\
2015\end{array}$ & 135 & Single & Elective & Intra-operative & Fluids & SW \\
\hline Davies, 2019 & 241 & Multi & Emergency & Intra-operative & Fluids & SV \\
\hline Donati, 2007 & 135 & Multi & Elective & Intra/post-operative & Fluids \& IN & $\mathrm{O}_{2} \mathrm{ER}$ \\
\hline El-Sharkawy, 2013 & 59 & Single & Elective & Intra/post-operative & Fluids & SV, FTC \\
\hline Funk, 2015 & 40 & Single & Elective & Intra-operative & Fluids \& IN & $\mathrm{SW}, \mathrm{Cl}$ \\
\hline Gan, 2002 & 100 & Single & Elective & Intra-operative & Fluids & SV, FTC \\
\hline Gerent, 2018 & 128 & Single & Elective & Post-operative & Fluids \& IN & $\mathrm{SVI}, \mathrm{Cl}$ \\
\hline Goepfert, 2013 & 100 & Single & Elective & Intra/post-operative & Fluids \& IN & $\begin{array}{l}\text { GEDI, SW, } \\
\mathrm{Cl}\end{array}$ \\
\hline $\begin{array}{l}\text { Gómez-Izquierdo, } \\
2017\end{array}$ & 135 & Single & Elective & Intra-operative & Fluids & SV \\
\hline Han, 2016 & 40 & Single & Elective & Intra-operative & Fluids & SW \\
\hline Jammer, 2010 & 241 & Multi & Elective & Intra/post-operative & Fluids & $\mathrm{ScVO}_{2}$ \\
\hline Jammer, 2015 & 30 & Multi & Elective & Intra-operative & Fluids \& IN & SW \\
\hline Joosten, 2019 & 39 & Single & Elective & Intra-operative & Fluids & $\mathrm{Cl}, \mathrm{SW}$ \\
\hline Kaufmann, 2017 & 100 & Single & Elective & Intra-operative & Fluids \& IN & $\mathrm{SV}, \mathrm{Cl}$ \\
\hline Kaufmann, 2018 & 90 & Single & Mixed & Intra-operative & Fluids \& IN & $\mathrm{SV}, \mathrm{Cl}$ \\
\hline Kim, 2018 & 62 & Single & Elective & Intra-operative & Fluids \& IN & $\mathrm{SW}, \mathrm{Cl}$ \\
\hline Kumar, 2016 & 60 & Single & Elective & Intra-operative & Fluids \& IN & SW, SVR \\
\hline Lobo, 2000 & 37 & Single & Elective & Intra/post-operative & Fluids \& IN & $\mathrm{DO}_{2}, \mathrm{PAOP}$ \\
\hline Luo, 2017 & 150 & Single & Elective & Intra-operative & Fluids \& IN & $\mathrm{SW}, \mathrm{Cl}$ \\
\hline Mayer, 2010 & 60 & Single & Elective & Intra-operative & Fluids \& IN & $\mathrm{Cl}, \mathrm{SVl}$ \\
\hline McKendry, 2004 & 179 & Single & Mixed & Post-operative & Fluids \& IN & $\mathrm{SI}$ \\
\hline McKenny, 2013 & 102 & Single & Elective & Intra-operative & Fluids & SV \\
\hline Mikor, 2015 & 84 & Single & Elective & Intra-operative & Fluids \& IN & $\mathrm{ScVO}_{2}$ \\
\hline Moppett, 2015 & 130 & Single & Emergency & Intra-operative & Fluids & SV \\
\hline Mythen, 1995 & 60 & Single & Elective & Intra-operative & Fluids & SV, CVP \\
\hline Osawa, 2016 & 126 & Single & Elective & Intra/post-operative & Fluids \& IN & $\mathrm{SVI}, \mathrm{Cl}$ \\
\hline Pearse, 2005 & 122 & Single & Mixed & Post-operative & Fluids \& IN & $\mathrm{SV}, \mathrm{DO}_{2} \mathrm{l}, \mathrm{Cl}$ \\
\hline Pearse, 2014 & 734 & Multi & Mixed & Intra/post-operative & Fluids \& IN & SV \\
\hline
\end{tabular}


Table 2 Summary characteristics of included studies. (Continued)

\begin{tabular}{|c|c|c|c|c|c|c|}
\hline Author/year & $\begin{array}{l}\text { No. of } \\
\text { patients }\end{array}$ & $\begin{array}{l}\text { Single/ } \\
\text { multicenter }\end{array}$ & $\begin{array}{l}\text { Type of } \\
\text { surgery }\end{array}$ & Timing & $\begin{array}{l}\text { Fluids/fluids } \& \text { inotropes (I) or } \\
\text { vasopressors (V) }\end{array}$ & Goals \\
\hline Peng, 2014 & 80 & Single & Elective & Intra-operative & Fluids & SW \\
\hline Pestaña, 2014 & 170 & Multi & Elective & Intra/post-operative & Fluids \& IN & SV, Cl, MAP \\
\hline Phan, 2014 & 100 & Single & Elective & Intra-operative & Fluids & SVI, FTC \\
\hline Reisinger, 2017 & 58 & Single & Elective & Intra/post-operative & Fluids & SVI \\
\hline Salzwedel, 2013 & 180 & Multi & Elective & Intra-operative & Fluids \& IN & PPV, Cl \\
\hline Sandham, 2003 & 1994 & Multi & Mixed & Pre-operative & Fluids \& IN & $\mathrm{DO}_{2} \mathrm{l}, \mathrm{Cl}$ \\
\hline Scheeren, 2013 & 64 & Multi & Elective & Intra-operative & Fluids & SW, SV \\
\hline Schmid, 2016 & 193 & Single & Elective & Intra/post-operative & Fluids \& IN & GEDI, Cl \\
\hline Senagore, 2009 & 64 & Single & Elective & Intra-operative & Fluids & SV \\
\hline Shoemaker, 1998 & 88 & Single & Mixed & Pre-operative & Fluids \& IN & $\begin{array}{l}\mathrm{Cl}, \mathrm{DO}_{2} \mathrm{l} \\
\mathrm{VO}_{2} \mathrm{I}\end{array}$ \\
\hline Sinclair, 1997 & 40 & Single & Emergency & Intra-operative & Fluids & SV, FTC \\
\hline Stens, 2017 & 244 & Multi & Elective & Intra-operative & Fluids \& IN & $\mathrm{Cl}, \mathrm{PPV}$ \\
\hline Ueno, 1998 & 34 & Single & Elective & Post-operative & Fluids \& IN & $\begin{array}{l}\mathrm{Cl}, \mathrm{DO}_{2} \mathrm{l} \\
\text { VO2l }\end{array}$ \\
\hline Valentine, 1998 & 120 & Single & Elective & Pre-operative & Fluids \& IN & $\begin{array}{l}\text { PCWP, Cl, } \\
\text { SVR }\end{array}$ \\
\hline $\begin{array}{l}\text { Van der Linden, } \\
2010\end{array}$ & 57 & Single & Elective & Intra-operative & Fluids \& IN & $\mathrm{Cl}$ \\
\hline Venn, 2002 & 90 & Single & Emergency & Intra-operative & Fluids & SV, FTC \\
\hline Wakeling, 2005 & 134 & Single & Elective & Intra-operative & Fluids & SV \\
\hline Weinberg, 2017 & 52 & Multi & Elective & Intra-operative & Fluids \& IN & SW, Cl \\
\hline Weinberg, 2019 & 50 & Multi & Elective & Intra-operative & Fluids \& IN & SW, Cl \\
\hline Wilson, 1999 & 138 & Single & Elective & $\begin{array}{l}\text { Pre/intra/post- } \\
\text { operative }\end{array}$ & Fluids \& IN & $\mathrm{DO}_{2} \mathrm{I}$ \\
\hline Wu, 2017 & 66 & Single & Elective & Intra-operative & Fluids \& IN & SW, Cl \\
\hline Xu, 2017 & 172 & Single & Elective & Intra-operative & Fluids \& IN & SW, Cl \\
\hline Yin, 2018 & 50 & Single & Elective & Intra-operative & Fluids \& IN & SW, Cl \\
\hline Zakhaleva 2013 & 91 & Single & Elective & Intra-operative & Fluids & SV, FTC \\
\hline Zeng, 2014 & 60 & Single & Elective & Intra-operative & Fluids & SW \\
\hline Zhang, 2013 & 60 & Single & Elective & Intra-operative & Fluids \& IN & SW, Cl \\
\hline
\end{tabular}

Venn et al., 2002; Wakeling et al., 2005; Zakhaleva et al., 2013), pulmonary artery catheter (8 studies)(Bender et al., 1997; Boyd et al., 1993; Lobo et al., 2000; Sandham et al., 2003; Shoemaker et al., 1988; Ueno et al., 1998; Valentine et al., 1998; Wilson et al., 1999), non-invasive cardiac output monitor (5 studies)(Davies et al., 2019; Joosten et al., 2019; Pestana et al., 2014; Stens et al., 2017; Yin et al., 2018) and those measuring oxygen parameters (3 studies)(Donati et al., 2007; Jammer et al., 2010; Mikor et al., 2015). Goal-directed haemodynamic therapy was initiated intra-operatively in the majority of studies (82\%)(Bahlmann et al., 2019; Benes et al., 2010; Bisgaard et al., 2013a; Bisgaard et al., 2013b; Brandstrup et al., 2012; Calvo-Vecino et al., 2018; Cecconi et al., 2011; Colantonio et al., 2015; Correa-Gallego et al., 2015; Davies et al., 2019; Donati et al., 2007; El Sharkawy et al., 2013; Funk et al., 2015; Gan et al., 2002; Goepfert et al., 2013; Gomez-Izquierdo et al., 2017; Han et al., 2016; Jammer et al., 2010; Jammer et al., 2015; Joosten et al., 2019; Kaufmann et al., 2017; Kaufmann et al., 2018; Kim et al., 2018; Kumar et al., 2016; Lobo et al., 2000; Luo et al., 2017; Mayer et al., 2010; McKenny et al., 2013; Mikor et al., 2015; Moppett et al., 2015; Mythen \& Webb, 1995; Osawa et al., 2016; Pearse et al., 2014; Peng et al., 2014; Pestana et al., 2014; Phan et al., 2014; Reisinger et al., 2017; Salzwedel et al., 2013; Scheeren et al., 2013; Schmid et al., 2016; Senagore et al., 2009; Sinclair et al., 1997; Stens et al., 2017; Van der Linden et al., 2010; Venn et al., 2002; Wakeling et al., 2005; Weinberg et al., 2017; Weinberg et al., 2019; Wu et al., 2017; Xu et al., 2017; Yin et al., 2018; Zakhaleva et al., 2013; Zeng et al., 2014; Zhang et al., 2013). In 22 
studies (33\%), the GDHT intervention used fluids alone(Brandstrup et al., 2012; Correa-Gallego et al., 2015; El Sharkawy et al., 2013; Gan et al., 2002; GomezIzquierdo et al., 2017; Han et al., 2016; Jammer et al., 2010; Joosten et al., 2019; Kim et al., 2018; McKenny et al., 2013; Moppett et al., 2015; Mythen \& Webb, 1995; Peng et al., 2014; Phan et al., 2014; Reisinger et al., 2017; Scheeren et al., 2013; Senagore et al., 2009; Sinclair et al., 1997; Venn et al., 2002; Wakeling et al., 2005; Zakhaleva et al., 2013; Zeng et al., 2014) and the rest used fluids with vasoactive or inotropic agents as part of their GDHT protocol(Ackland et al., 2015; Bahlmann et al., 2019; Bartha et al., 2013; Bender et al., 1997; Benes et al., 2010; Bisgaard et al., 2013a; Bisgaard et al., 2013b; Boyd et al., 1993; Calvo-Vecino et al., 2018; Cecconi et al., 2011; Colantonio et al., 2015; Davies et al., 2019; Donati et al., 2007; Funk et al., 2015; Gerent et al., 2018; Goepfert et al., 2013; Jammer et al., 2015; Kaufmann et al., 2017; Kaufmann et al., 2018; Kim et al., 2018; Lobo et al., 2000; Luo et al., 2017; Mayer et al., 2010; McKendry et al., 2004; Mikor et al., 2015; Osawa et al., 2016; Pearse et al., 2005; Pearse et al., 2014; Pestana et al., 2014; Salzwedel et al., 2013; Sandham et al., 2003; Schmid et al., 2016; Shoemaker et al., 1988; Stens et al., 2017; Ueno et al., 1998; Valentine et al., 1998; Van der Linden et al., 2010; Weinberg et al., 2017; Weinberg et al., 2019; Wilson et al., 1999; Wu et al., 2017; Xu et al., 2017; Yin et al., 2018; Zhang et al., 2013). Among those which used inotropes, more than $50 \%$ used dobutamine(Ackland et al., 2015; Bahlmann et al., 2019; Bartha et al., 2013; Benes et al., 2010; Bisgaard et al., 2013a; Bisgaard et al., 2013b; Cecconi et al., 2011; Donati et al., 2007; Gerent et al., 2018; Kim et al., 2018; Kumar et al., 2016; Lobo et al., 2000; Mayer et al., 2010; Osawa et al., 2016; Pestana et al., 2014; Salzwedel et al., 2013; Schmid et al., 2016; Shoemaker et al., 1988; Stens et al., 2017; Ueno et al., 1998; Van der Linden et al., 2010; Wu et al., 2017; Xu et al., 2017; Yin et al., 2018; Zhang et al., 2013). The fluid bolus in the GDHT algorithm consisted of a colloid solution in nearly two-thirds of the studies (Cecconi et al., 2011; Chappell et al., 2008; Chong et al., 2018; Correa-Gallego et al., 2015; Davies et al., 2019; Donati et al., 2007; Eappen et al., 2013; El Sharkawy et al., 2013; Evans et al., 2009; Funk et al., 2015; Gerent et al., 2018; Goepfert et al., 2013; Gomez-Izquierdo et al., 2017; Grocott et al., 2012; Guyatt et al., 2008; Hamilton et al., 2011; Han et al., 2016; Higgins \& Green, 2011; Joosten et al., 2019; Kim et al., 2018; Lobo et al., 2000; Luo et al., 2017; Mayer et al., 2010; McKendry et al., 2004; McKenny et al., 2013; Moppett et al., 2015; Mythen \& Webb, 1995; Noblett et al., 2006; Osawa et al., 2016; Pearse et al., 2005; Pearse et al., 2014; Pestana et al., 2014; Phan et al., 2014; Ruscic et al., 2017; Schmid et al., 2016; Senagore et al., 2009; Shoemaker et al., 1988; Ueno et al., 1998; Valentine et al., 1998; Van der Linden et al., 2010; Wakeling et al., 2005; Weinberg et al., 2017). Nine studies used a combination of crystalloids and colloids(Calvo-Vecino et al., 2018; Gerent et al., 2018; Kumar et al., 2016; Lobo et al., 2000; Mayer et al., 2010; Senagore et al., 2009; Shoemaker et al., 1988; Stens et al., 2017; Wilson et al., 1999) and a further 9 studies used crystalloids alone for fluid boluses as part of their GDHT protocol (Colantonio et al., 2015; Gan et al., 2002; http://www.isrctn.com/ISRCTN14729158, n.d.; Jammer et al., 2015; Jammer et al., 2010; Jhanji et al., 2010; Mikor et al., 2015; Scheeren et al., 2013; Sinclair et al., 1997).

All included studies were assessed for the risk of bias using the Cochrane Collaboration tool for assessing risk of bias.(Higgins \& Green, 2011) Each study was assessed according to seven domains: random sequence generation, allocation concealment, blinding of participants and personnel, blinding of outcome assessment, incomplete outcome data, selective reporting and other biases. Random sequence generation was identified as at low risk of bias in 45 studies (68\%) (Cecconi et al., 2011; Chappell et al., 2008; Chong et al., 2018; Correa-Gallego et al., 2015; Davies et al., 2019; Donati et al., 2007; Eappen et al., 2013; Edwards et al., 2019; Evans et al., 2009; Grocott et al., 2012; Grocott et al., 2005; Guyatt et al., 2008; Hamilton et al., 2011; Higgins \& Green, 2011; http:// www.isrctn.com/ISRCTN14729158, n.d.; Jammer et al., 2015; Jammer et al., 2010; Jhanji et al., 2010; Joosten et al., 2019; Kim et al., 2018; Lobo et al., 2000; McKendry et al., 2004; Mikor et al., 2015; Moppett et al., 2015; Mythen \& Webb, 1995; Noblett et al., 2006; Osawa et al., 2016; Pearse et al., 2005; Pearse et al., 2014; Peng et al., 2014; Phan et al., 2014; Polonen et al., 2000; Reisinger et al., 2017; Sandham et al., 2003; Schmid et al., 2016; Senagore et al., 2009; Sinclair et al., 1997; Srinivasa et al., 2013; Stens et al., 2017; Valentine et al., 1998; Van der Linden et al., 2010; Venn et al., 2002). Allocation concealment was judged to be adequate in 57 studies (88\%)(Ackland et al., 2015; Bahlmann et al., 2019; Bartha et al., 2013; Benes et al., 2010; Brandstrup et al., 2012; CalvoVecino et al., 2018; Cecconi et al., 2011; Colantonio et al., 2015; Correa-Gallego et al., 2015; Davies et al., 2019; El Sharkawy et al., 2013; Funk et al., 2015; Gan et al., 2002; Gerent et al., 2018; Goepfert et al., 2013; Gomez-Izquierdo et al., 2017; Jammer et al., 2010; Jammer et al., 2015; Joosten et al., 2019; Kaufmann et al., 2017; Kaufmann et al., 2018; Kumar et al., 2016; Lobo et al., 2000; Luo et al., 2017; Mayer et al., 2010; McKendry et al., 2004; McKenny et al., 2013; Mikor et al., 2015; Moppett et al., 2015; Mythen \& Webb, 1995; Osawa et al., 2016; Pearse et al., 2005; 
Pearse et al., 2014; Peng et al., 2014; Pestana et al., 2014; Phan et al., 2014; Reisinger et al., 2017; Salzwedel et al., 2013; Sandham et al., 2003; Scheeren et al., 2013; Schmid et al., 2016; Senagore et al., 2009; Shoemaker et al., 1988; Sinclair et al., 1997; Stens et al., 2017; Valentine et al., 1998; Van der Linden et al., 2010; Venn et al., 2002; Wakeling et al., 2005; Weinberg et al., 2017; Weinberg et al., 2019; Wilson et al., 1999; Wu et al., 2017; Xu et al., 2017; Yin et al., 2018; Zakhaleva et al., 2013; Zhang et al., 2013). Blinding of participants and personnel was achieved adequately in only 25 studies (38\%) (Cecconi et al., 2011; Correa-Gallego et al., 2015; Eappen et al., 2013; Edwards et al., 2019; El Sharkawy et al., 2013; Gan et al., 2002; Hamilton et al., 2011; Jammer et al., 2010; Jhanji et al., 2010; Lobo et al., 2000; McKendry et al., 2004; Moppett et al., 2015; Noblett et al., 2006; Osawa et al., 2016; Pearse et al., 2005; Pearse et al., 2014; Polonen et al., 2000; Ruscic et al., 2017; Schmid et al., 2016; Senagore et al., 2009; Shoemaker et al., 1988; Srinivasa et al., 2013; Ueno et al., 1998; Valentine et al., 1998; Van der Linden et al., 2010), and blinding of outcome in 38 (58\%)(Ackland et al., 2015; Benes et al., 2010; Bisgaard et al., 2013a; Bisgaard et al., 2013b; Brandstrup et al., 2012; Calvo-Vecino et al., 2018; Colantonio et al., 2015; Correa-Gallego et al., 2015; Davies et al., 2019; Funk et al., 2015; Gan et al., 2002; Gerent et al., 2018; Goepfert et al., 2013; Gomez-Izquierdo et al., 2017; Jammer et al., 2010; Jammer et al., 2015; Joosten et al., 2019; Kaufmann et al., 2017; Kaufmann et al., 2018; Kim et al., 2018; Mayer et al., 2010; McKendry et al., 2004; McKenny et al., 2013; Moppett et al., 2015; Osawa et al., 2016; Pearse et al., 2005; Pearse et al., 2014; Peng et al., 2014; Pestana et al., 2014; Phan et al., 2014; Reisinger et al., 2017; Sandham et al., 2003; Scheeren et al., 2013; Van der Linden et al., 2010; Weinberg et al., 2017; Wu et al., 2017; Xu et al., 2017; Zeng et al.,
2014). In nine studies (14\%), there was incomplete outcome data(El Sharkawy et al., 2013; Jammer et al., 2015; Pestana et al., 2014; Salzwedel et al., 2013; Scheeren et al., 2013; Stens et al., 2017; Zakhaleva et al., 2013; Zeng et al., 2014; Zhang et al., 2013). Sixty-one studies (92\%) were identified as at low risk of selective reporting bias(Ackland et al., 2015; Bahlmann et al., 2019; Bartha et al., 2013; Bender et al., 1997; Benes et al., 2010; Bisgaard et al., 2013a; Bisgaard et al., 2013b; Boyd et al., 1993; Brandstrup et al., 2012; Calvo-Vecino et al., 2018; Cecconi et al., 2011; Colantonio et al., 2015; Correa-Gallego et al., 2015; Davies et al., 2019; Donati et al., 2007; Funk et al., 2015; Gan et al., 2002; Gerent et al., 2018; Goepfert et al., 2013; Gomez-Izquierdo et al., 2017; Jammer et al., 2010; Joosten et al., 2019; Kaufmann et al., 2017; Kaufmann et al., 2018; Kim et al., 2018; Kumar et al., 2016; Luo et al., 2017; Mayer et al., 2010; McKendry et al., 2004; McKenny et al., 2013; Mikor et al., 2015; Moppett et al., 2015; Mythen \& Webb, 1995; Osawa et al., 2016; Pearse et al., 2005; Pearse et al., 2014; Peng et al., 2014; Pestana et al., 2014; Phan et al., 2014; Reisinger et al., 2017; Salzwedel et al., 2013; Sandham et al., 2003; Scheeren et al., 2013; Schmid et al., 2016; Senagore et al., 2009; Shoemaker et al., 1988; Sinclair et al., 1997; Stens et al., 2017; Valentine et al., 1998; Van der Linden et al., 2010; Venn et al., 2002; Wakeling et al., 2005; Weinberg et al., 2017; Weinberg et al., 2019; Wilson et al., 1999; Wu et al., 2017; Xu et al., 2017; Yin et al., 2018; Zakhaleva et al., 2013; Zeng et al., 2014; Zhang et al., 2013). Multiple studies reported industry funding, which was considered a potential source of bias. In one study, the second author has been investigated for scientific misconduct(Mayer et al., 2010). The overall risk of bias is presented in Fig. 2 and a table of individual study bias is available in the Supplementary material (Figure A).

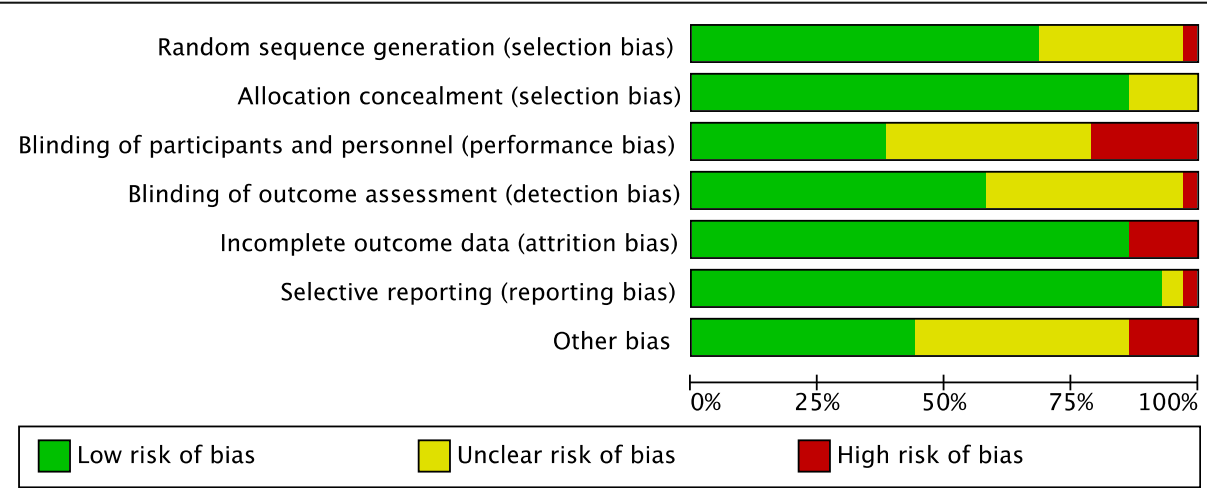

Fig. 2 Risk of bias graph: review authors' judgements about each risk of bias item presented as percentages across all included studies. Green indicates no risk of bias, yellow and red represent unclear risk and high risk respectively. 


\section{Synthesis of results Primary outcome}

Sixty-six studies with a total of 9548 participants reported post-operative pulmonary events. These respiratory events accounted for $11.5 \%(549 / 4772)$ of the GDHT group and 14\% (676/4776) of the control group. There was a significant difference between groups (OR $0.74,95 \%$ CI 0.59 to $0.92 ; I^{2}=47 \%, P=0.007$ ) (Fig. 3). A random-effects model was utilised as there was moderate statistical heterogeneity. There was substantial clinical heterogeneity among the included studies with variations in age of study (1988 to 2019), study population, methodology, operative and GDHT interventions and measured outcomes. The quality of evidence was downgraded to moderate due to statistical and clinical heterogeneity. Funnel plot analysis suggested visually no significant asymmetry with Egger's regression intercept of -0.1046 (95\% CI -0.7659 to 0.5567$)$ and $P=0.7530$ (Fig. 4).

\section{Secondary outcomes}

We assessed individual respiratory outcomes such as pulmonary infections, ARDS, ALI, pulmonary embolism, and pulmonary oedema as secondary outcomes. Goal-directed haemodynamic therapy significantly reduced the incidence of post-operative respiratory tract infections or pneumonia (OR 0.72 [95\% 0.60 to 0.86], $I^{2}=0 \%, P=0.0003,45$ studies) and pulmonary oedema (OR 0.47 [ $95 \%$ CI 0.30 to 0.73 ], $I^{2}=0 \%, P$ $=0.0008,23$ studies). However, there were no differences in ARDS (OR 0.57 [95\% CI 0.31 to 1.02], $I^{2}=$ $17 \%, P=0.06,15$ studies) or pulmonary embolism (OR 1.08 [95\% CI 0.59 to 1.95 ], $I^{2}=1 \%, P=0.81,28$ studies) (Table 3).

The intra-operative fluid input and balance was presented in a number of different ways by the included studies. Overall, sixty-two studies presented fluid data, from which intra-operative fluid data was available in 56 studies. However, 4 studies presented these as a rate in $\mathrm{ml} / \mathrm{kg} / \mathrm{h}$ and we were therefore not able to use these for data synthesis. Forty-two studies presented the intra-operative fluid input data separately for colloids and crystalloids. In the GDHT group, there were less crystalloids $(-375 \mathrm{ml}$ [95\% CI -581 to $\left.-170], I^{2}=98 \%, P=0.0003\right)$ and more colloids ( $+281 \mathrm{ml}$ [95\% CI 166 to 396], $I^{2}=96 \%, P<0.00001$ ) administered intra-operatively. There was significant statistical heterogeneity between studies and randomeffects models were used for these analyses. Total intra-operative fluid input was presented by 27 studies and the GDHT group overall had less cumulative intra-operative fluid $(-212 \mathrm{ml}$ [95\% CI -254 to 171], $\left.I^{2}=91 \%, P<0.0001\right)$ than controls (Table 4$)$.

\section{Subgroup analysis}

We carried out a subgroup analysis to assess the impact of GDHT on the incidence of pulmonary complications adjusted for the type of surgery (Table 5). There was a beneficial effect demonstrated for patients undergoing general, mixed or abdominal surgery (OR 0.76 [95\% CI $0.58-0.99], I^{2}=54 \%, P=$ $0.04,44$ studies) and cardiothoracic surgery (OR 0.33 [95\% CI 0.17 to 0.63 ], $I^{2}=22 \%, P=0.0009,7$ studies). No reduction in pulmonary complications was observed in patients undergoing orthopaedic (OR 1.15 [95\% CI 0.73 to 1.81 ], $I^{2}=0 \%, P=0.54,9$ studies) or vascular surgery (OR 0.79 [95\% CI 0.35 to 1.76$], I^{2}=$ $12 \%, P=0.56,6$ studies).

Each study was also categorised according to their method of monitoring. Most studies (50\%) used minimally invasive cardiac output monitoring and the results were not significant for any specific category of technique (Table 5).

The GDHT protocol used fluids only in 22 studies. Other studies used a combination of fluid bolus with inotropes and/or vasopressors. Forty-two studies used colloids as their bolus fluid and 9 studies used crystalloids, and a further 9 studies used a combination of both crystalloids and colloids. When studies used fluids in combination with inotropes or vasopressors in the GDHT protocol, there was a significant reduction in pulmonary complications (OR 0.62 [95\% CI 0.49 to $0.80], I^{2}=50 \%, P=0.0002$, 44 studies). This was not evident when the protocol used fluid alone (OR 1.39 [95\% CI 0.89 to 2.16], $I^{2}=17 \%, \mathrm{P}=0.14,22$ studies) (Table 5). Given the significant advances in perioperative care over the inclusion period of this meta-analysis, we compared the effect of GDHT on pulmonary complications with dates when the studies were conducted. We grouped these trials into 3 cohorts: year 2000 and earlier (OR 0.54 [95\% CI 0.36 to 0.81 ], $I^{2}=0 \%, P=0.003,9$ studies), 2001 to 2010 (OR 0.86 [95\% CI 0.56 to 1.31 ], $I^{2}$ $=42 \%, P=48,12$ studies), and 2011 to the current period (OR 0.73 [95\% CI 0.54 to 0.99], $I^{2}=53 \%, P=$ $0.04,45$ studies). Most of the studies were conducted within the current decade (Table 5).

\section{Discussion}

This systematic review and meta-analysis identified 96 studies evaluating the effect of goal-directed fluid therapy, utilising various devices, to assess patients' outcomes in the perioperative setting. Sixty-six studies with 9548 participants were included in the data synthesis. The overall incidence of pulmonary complication was $13 \%$ and, based on moderate-quality evidence, this review shows that peri-operative goaldirected haemodynamic therapy, targeted to augment blood flow by specified measured goals, reduces 


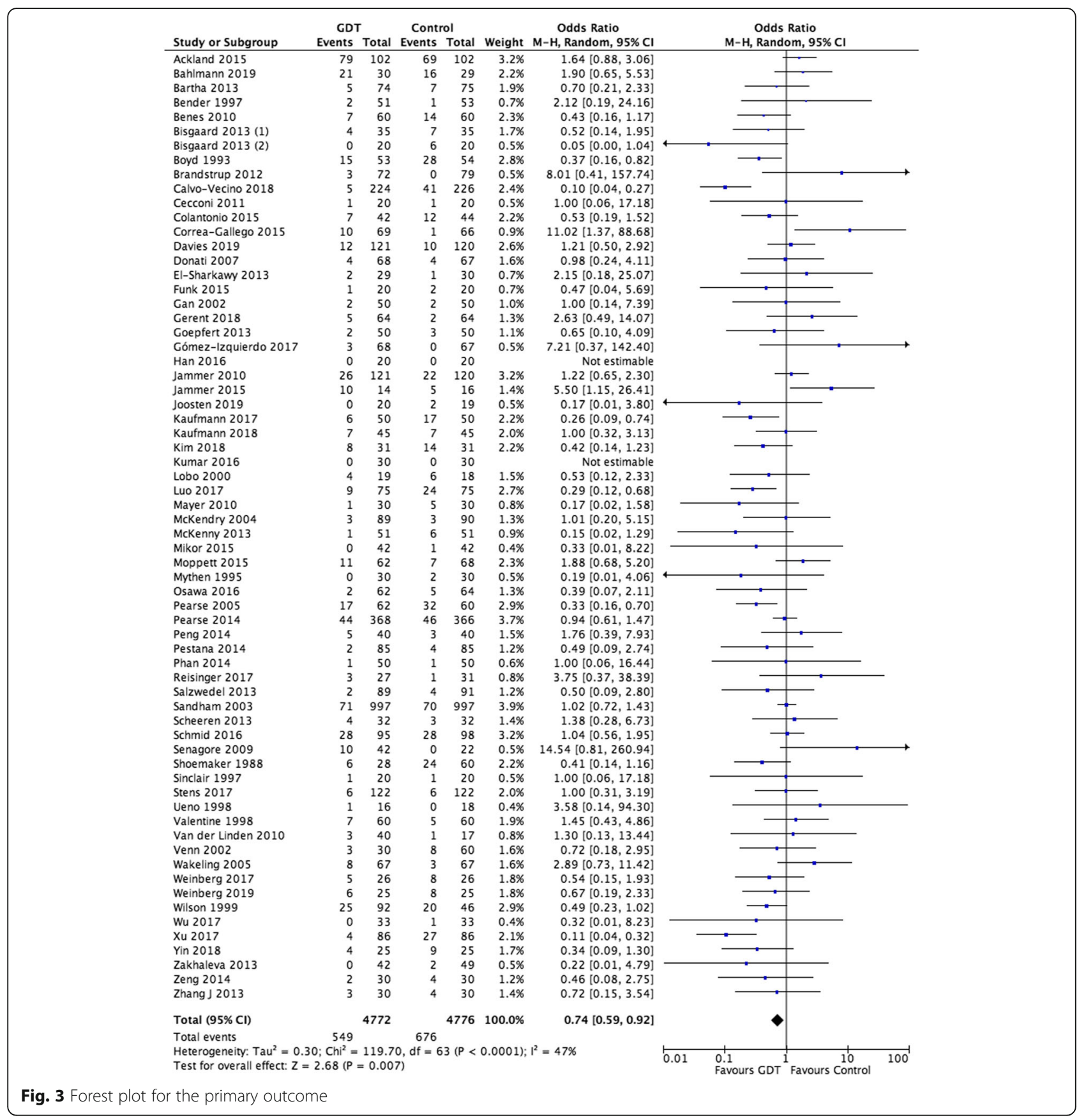

pulmonary complications. The results are limited by the presence of substantial clinical and moderate statistical heterogeneity. Consequently, we have downgraded the GRADE evidence from high to moderate. This is the first systematic review to assess the impact of goal-directed haemodynamic therapy exclusively on post-operative pulmonary complications in patients undergoing surgery.

The rates of pulmonary infection and pulmonary oedema were significantly lower in the intervention group with a number needed to treat 45 and 48 respectively. Although the data synthesis was limited by publication bias, where only a proportion of published studies reported specific complication rates (pulmonary infections $68 \%$; pulmonary oedema $35 \%$; pulmonary embolism 42\%; and ARDS 23\%), these findings are consistent with previously published systematic reviews in this area.(Grocott et al., 2012; Chong et al., 2018) To mitigate clinical heterogeneity, we performed subgroup analysis for types of surgery, devices utilised and the GDHT interventions (fluids alone or fluids and inotropes or vasopressors) provided. The beneficial effect was 


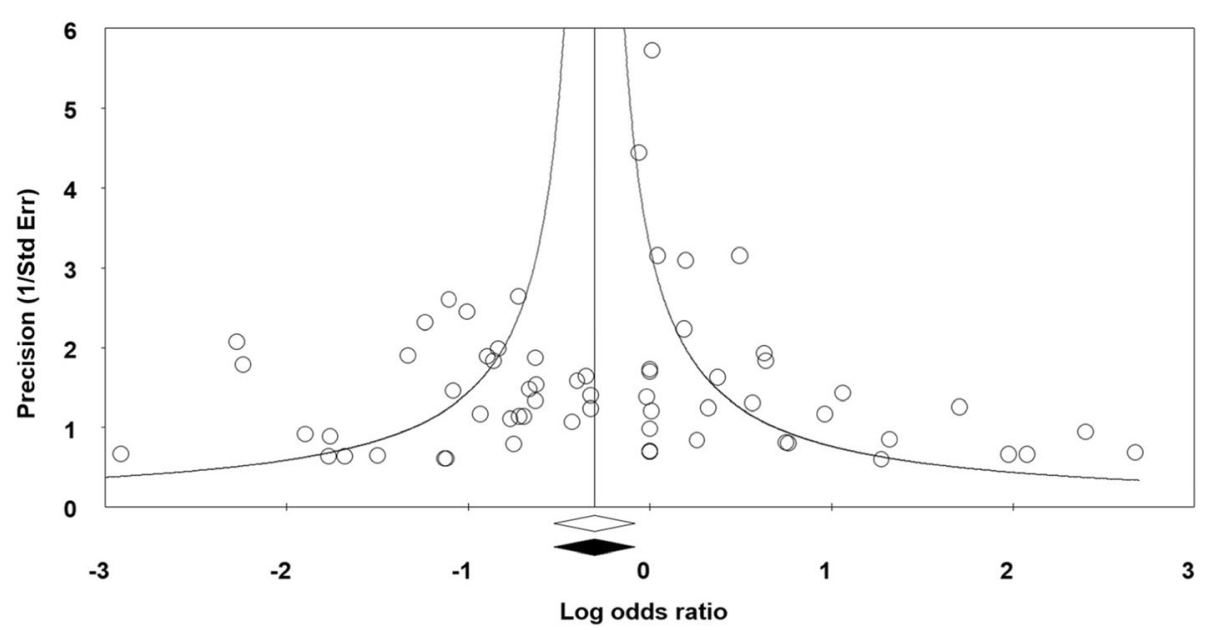

Fig. 4 Funnel plot of precision by log odds ratio

demonstrated in patients undergoing cardiothoracic and major abdominal surgery and was not specific to any device category. The GDHT algorithm with a combination of fluids and vasopressors or inotropes was associated with a significant reduction in post-operative pulmonary complications, whilst no benefit was demonstrated for those using fluids alone.

Although GDHT may impact pulmonary complications through a variety of mechanisms relating to the monitoring device, type of fluid, different combinations of vasopressor and/or inotrope therapy, we found no evidence that this intervention was harmful. The underlying reasons for the demonstrated beneficial effects are not evaluated by this review. However, several possible mechanisms may explain these findings. A GDHT algorithm with a combination of fluids and inotropes or vasopressors seems to have a positive effect on the incidence of pulmonary complications compared with fluid therapy alone. Additionally, the GDHT participants had less crystalloid $(380 \mathrm{ml})$ and more colloid solutions $(280 \mathrm{ml})$ intra-operatively. Whilst these differences are modest, they may in combination have contributed to maintaining intravascular volume, whilst reducing the development of tissue and pulmonary interstitial oedema. The occurrence of a beneficial outcome in patients receiving more colloid during surgery challenges notions that the use of such fluids has no benefit (Chappell et al., 2008). Moreover, goal-directed haemodynamic therapy has been shown to improve gut perfusion(Mythen \& Webb, 1995) and mitigate the inflammatory response to surgery(Noblett et al., 2006) as well as improving tissue microvascular flow and oxygenation(Jhanji et al., 2010). Overall, improved tissue perfusion as a result of GDHT may reduce systematic inflammation and thereby minimise secondary pulmonary harm. Future large randomised controlled trials may offer additional information regarding the use of this intervention in emergency and major gastrointestinal surgical patients (ISRCTN14729158 and ISRCTN39653756, Table 1).

Our review has several limitations. We were not able to include all published studies in this area due to a lack of consistent reporting of pulmonary complications. Recent efforts to standardise core outcome sets may improve this situation in the future.(Boney et al., 2016) Furthermore, there was substantial clinical heterogeneity among the studies included in this review. The studies have varied in nature with regard to type and mode of surgery (elective or emergency), devices, protocols and interventions utilised for the goal-directed pathway and were conducted over several decades, during which surgical and anaesthetic practice has evolved substantially (e.g. introduction of laparoscopic surgery and enhanced

Table 3 Secondary outcome for specific pulmonary complications. ARDS acute respiratory distress syndrome, Cl confidence interval, M-H Mantel-Haenszel

\begin{tabular}{llllll}
\hline Outcome & No. of studies & No. of patients & Analysis & Effect $(\mathbf{9 5} \%$ Cl) & $P$ value \\
\hline Chest infection or pneumonia & 45 & 6969 & M-H odds ratio & $0.72(0.60-0.86)$ & $P=0.0003$ \\
ARDS & 15 & 2491 & M-H odds ratio & $0.57(0.31-1.02)$ & $P=0.06$ \\
Pulmonary oedema & 23 & 3205 & M-H odds ratio & $0.47(0.30-0.73)$ & $P=0.0008$ \\
Pulmonary embolism & 28 & 5430 & M-H odds ratio & $1.08(0.59-1.95)$ & $P=0.81$ \\
\hline
\end{tabular}


Table 4 Intraoperative fluid balance. The effect was presented as a mean difference in goal-directed haemodynamic group in comparison with controls. Cl confidence interval, IV inverse variance

\begin{tabular}{llllll}
\hline Outcome & No. of studies & No. of patients & Analysis & Effect $(\mathbf{9 5 \%}$ Cl) & $P$ value \\
\hline Intra-operative crystalloids & 42 & 4956 & Mean difference (IV, random) & $-375(-581$ to -170$)$ & $P=0.0003$ \\
Intra-operative colloids & 42 & 4984 & Mean difference (IV, random) & $281(166$ to 396) & $P<0.00001$ \\
Cumulative intra-operative fluid input & 27 & 2907 & Mean difference (IV, random) & $-212(-254$ to 171) & $P<0.00001$ \\
\hline
\end{tabular}

recovery). Some studies were conducted in high-risk groups and others embedded enhanced recovery programmes (ERAS). We did not differentiate between these as they were not specifically reported by all studies. We have used author-defined definitions for pulmonary complications such as atelectasis, pulmonary infections or pneumonia, ALI and ARDS. Reporting of such definitions was variable between studies and may have introduced bias. We have included all patients with pulmonary oedema and whilst this was inclusive of patients with respiratory causes of pulmonary oedema it may have introduced additional bias from the inclusion of patients with cardiogenic pulmonary oedema. However, we did exclude studies that specifically mentioned pulmonary oedema of cardiogenic origin. Despite all these limitations, we have included as many of the studies as possible that reported pulmonary complications. We were also unable to include other robust clinical outcomes such as intensive care utilisation and the requirement for ventilatory support resulting from postoperative pulmonary complications due to a lack of consistent reporting. We have included both studies that augment haemodynamic variables to normal levels, as well as those that aimed to achieve supra-normal values of oxygen delivery. Recently, several studies have aimed to restrict fluid volume as part of their GDHT targets; however, we did not separate these in our data synthesis.

The paucity of clinical data and lack of consistent reporting of fluid administration among studies may have introduced publication bias, where not all the studies reporting the intra-operative fluid data in a format that we could analyse. As a result, we have performed data transformation to generate standard deviation and mean values from the median data presented (interquartile range, range, 95\% confidence intervals, and 25th and 75th percentiles), guided by the Cochrane Collaboration. Furthermore, most studies reported intra-operative fluid volumes separately for crystalloids and colloids. Some

Table 5 Subgroup analysis for the type of surgery, device, the goal-directed haemodynamic therapy intervention (fluids only or fluids and inotropes or vasopressors) and the date when the study was conducted. Cl confidence interval, $\mathrm{M}-\mathrm{H}$ Mantel-Haenszel, PAC pulmonary artery catheter

\begin{tabular}{|c|c|c|c|c|c|}
\hline Subgroups & No. of studies & No. of patients & Analysis & $\begin{array}{l}\text { Effect } \\
(95 \% \mathrm{Cl})\end{array}$ & $P$ value \\
\hline \multicolumn{6}{|l|}{ Surgery types } \\
\hline General/mixed/abdominal & 44 & 7420 & M-H odds ratio & $0.76(0.58-0.99)$ & $P=0.04$ \\
\hline Orthopaedic & 9 & 900 & M-H odds ratio & $1.15(0.73-1.81)$ & $P=0.54$ \\
\hline Cardiothoracic & 7 & 797 & M-H odds ratio & $0.33(0.17-0.63)$ & $P=0.0009$ \\
\hline Vascular & 6 & 431 & M-H odds ratio & $0.79(0.35-1.76)$ & $P=0.56$ \\
\hline \multicolumn{6}{|l|}{ Device types } \\
\hline Minimally invasive & 33 & 3719 & M-H odds ratio & $0.73(0.53-1.01)$ & $P=0.06$ \\
\hline Oesophageal doppler & 17 & 2003 & M-H odds ratio & $0.87(0.43-1.77)$ & $P=0.70$ \\
\hline Non-invasive & 5 & 744 & $\mathrm{M}-\mathrm{H}$ odds ratio & $0.77(0.44-1.38)$ & $P=0.38$ \\
\hline PAC & 8 & 2688 & M-H odds ratio & $0.69(0.44-1.08)$ & $P=0.10$ \\
\hline Oxygen indices & 3 & 460 & M-H odds ratio & $1.13(0.64-2.00)$ & $P=0.67$ \\
\hline \multicolumn{6}{|l|}{ Intervention per protocol } \\
\hline Fluids only & 22 & 2033 & M-H odds ratio & $1.39(0.89-2.16)$ & $P=0.14$ \\
\hline Fluid and inotropes or vasopressors & 44 & 7515 & M-H odds ratio & $0.62(0.49-0.80)$ & $P=0.0002$ \\
\hline \multicolumn{6}{|l|}{ Date of study publication } \\
\hline Conducted up to and year 2000 & 9 & 728 & M-H odds ratio & $0.54(0.36-0.81)$ & $P=0.003$ \\
\hline Conducted between 2001 and 2010 & 12 & 3296 & M-H odds ratio & $0.86(0.56-1.31)$ & $P=0.48$ \\
\hline Conducted between 2011 and to date & 45 & 5524 & M-H odds ratio & $0.73(0.54-0.99)$ & $P=0.04$ \\
\hline
\end{tabular}


studies reported these as $\mathrm{ml} / \mathrm{kg} / \mathrm{hour}$ and others as a cumulative balance, or total volumes used. Despite these limitations, it appears that in the GDHT group, more colloid and less crystalloid fluids were used during the intraoperative period. Most of the studies (63\%) utilised a colloid solution as their fluid of choice in their protocol for fluid bolus regimens and this is the likely explanation for the increased usage of colloids. Others used a combination of crystalloids and colloids (14\%), or crystalloids alone (14\%) and in six studies the type of fluid was not specified.

The risk of bias was an issue among the included studies. Many of the studies (62\%) included in this review did not blind the participants or personnel delivering the intervention peri-operatively and as a result, may have increased the risk of performance bias. Although some studies have attempted to blind but ultimately have found it difficult to fully blind the clinicians involved in delivering the therapy. Taking a pragmatic view, it is difficult to blind clinicians performing a continuous intervention that requires feedback and action in real-time and this has been taken into consideration when performing our risk of bias assessment and the interpretation of the results. Overall, the GRADE quality of evidence was graded as moderate due to substantial clinical and moderate statistical heterogeneity with a high risk of bias in several included studies.

Despite these limitations, the results of this systematic review and meta-analysis add to a growing body of literature suggesting that GDHT is safe and is effective in reducing the burden of a wide range of perioperative outcomes, in this case, respiratory complications. Whilst clinical adoption of GDHT is recognised to be variable, as the evidence base continues to grow the signal of benefit remains consistent. Understandable concerns about the evidence base are primarily focussed on the clinical heterogeneity, particularly the long duration of time over which these studies have taken place and the associated changes in clinical practice that have occurred, potentially limiting the validity of the findings for current practice. Results from two large ( $>2000 \mathrm{pa}$ tient) randomised controlled trials currently ongoing may clarify this picture soon.(Edwards et al., 2019; http://www.isrctn.com/ISRCTN14729158, n.d.)

\section{Conclusions}

This study has shown a reduction in post-operative pneumonia and pulmonary oedema following the use of GDHT. These beneficial effects were seen in studies using fluids in combination with inotropes or vasopressors in the GDHT protocol, but not when the protocol used fluid alone. Sub-group analyses demonstrated (i) benefit from GDHT in general/abdominal/mixed and cardiothoracic surgery but not in orthopaedic and vascular surgery; and (ii) no difference between devices used in the GDHT protocols. There was no demonstrable difference in the total volumes of fluid administered but there was greater use of colloid and less of crystalloid solutions in the GDHT group.

\section{Supplementary information}

Supplementary information accompanies this paper at https://doi.org/10. 1186/s13741-020-00161-5.

\section{Additional file 1: Supplementary material Table. A Study}

characteristics in detail. Supplementary material Figure A: review authors' judgements about each risk of bias item presented as percentages across all included studies. Green indicates no risk of bias, yellow and red represents unclear risk and high risk respectively

Ethics approval and consent for publication Not applicable.

\section{Authors' contributions}

$A D$ and $P R$ contributed to the study design. AD, MK, PR and MG conducted the study. AD, MK and PR contributed to data analysis: AD, MK, PR and MG contributed to writing paper: $A D, M K, P R, M G$ contributed to the revision of the paper. The author(s) read and approved the final manuscript.

\section{Funding}

No source of funding to be declared.

\section{Availability of data and materials}

The datasets during and/or analysed during the current study available from the corresponding author on reasonable request.

\section{Competing interests}

The authors declare that they have no competing interests.

\section{Author details}

${ }^{1}$ General Intensive Care Unit, University Hospital Southampton NHS Foundation Trust, Southampton SO16 6YD, UK. ${ }^{2}$ Anaesthesia Perioperative and Critical Care Research Group, Southampton NIHR Biomedical Research Centre, University Hospital Southampton/University of Southampton, Southampton, UK. ${ }^{3}$ Clinical and Experimental Sciences, Faculty of Medicine, University of Southampton, Southampton, UK.

Received: 15 June 2020 Accepted: 22 September 2020

Published online: 15 October 2020

\section{References}

Ackland GL, Iqbal S, Paredes LG, et al. Individualised oxygen delivery targeted haemodynamic therapy in high-risk surgical patients: a multicentre, randomised, double-blind, controlled, mechanistic trial. Lancet Respir Med. 2015;3:33-41.

Bahlmann H, Halldestam I, Nilsson L. Goal-directed therapy during transthoracic oesophageal resection does not improve outcome: Randomised controlled trial. Eur J Anaesthesiol. 2019;36:153-61.

Bartha E, Arfwedson C, Imnell A, Fernlund ME, Andersson LE, Kalman S. Randomized controlled trial of goal-directed haemodynamic treatment in patients with proximal femoral fracture. Br J Anaesth. 2013;110:545-53.

Bender JS, Smith-Meek MA, Jones CE. Routine pulmonary artery catheterization does not reduce morbidity and mortality of elective vascular surgery: results of a prospective, randomized trial. Ann Surg. 1997;226:229-36.

Benes J, Chytra I, Altmann P, et al. Intraoperative fluid optimization using stroke volume variation in high risk surgical patients: results of prospective randomized study. Crit Care. 2010;14:R118.

Bisgaard J, Gilsaa T, Ronholm E, Toft P. Optimising stroke volume and oxygen delivery in abdominal aortic surgery: a randomised controlled trial. Acta Anaesthesiol Scand. 2013a;57:178-88. 
Bisgaard J, Gilsaa T, Ronholm E, Toft P. Haemodynamic optimisation in lower limb arterial surgery: room for improvement? Acta Anaesthesiol Scand. 2013b;57:189-98

Boney O, Moonesinghe SR, Myles PS, Grocott MP. Standardizing endpoints in perioperative research. Can J Anaesth. 2016;63:159-68.

Boyd O, Grounds RM, Bennett ED. A randomized clinical trial of the effect of deliberate perioperative increase of oxygen delivery on mortality in high-risk surgical patients. JAMA. 1993;270:2699-707.

Brandstrup B, Svendsen PE, Rasmussen M, et al. Which goal for fluid therapy during colorectal surgery is followed by the best outcome: near-maximal stroke volume or zero fluid balance? Br J Anaesth. 2012;109:191-9.

Calvo-Vecino JM, Ripolles-Melchor J, Mythen MG, et al. Effect of goal-directed haemodynamic therapy on postoperative complications in low-moderate risk surgical patients: a multicentre randomised controlled trial (FEDORA trial). $\mathrm{Br}$ J Anaesth. 2018:120:734-44.

Canet J, Gallart L, Gomar C, et al. Prediction of postoperative pulmonary complications in a population-based surgical cohort. Anesthesiology. 2010; 113:1338-50

Cecconi M, Fasano N, Langiano N, et al. Goal-directed haemodynamic therapy during elective total hip arthroplasty under regional anaesthesia. Crit Care. 2011;15:R132.

Chappell D, Jacob M, Hofmann-Kiefer K, et al. A rational approach to perioperative fluid management. Anesthesiology. 2008;109:723-40.

Chong MA, Wang Y, Berbenetz NM, McConachie I. Does goal-directed haemodynamic and fluid therapy improve peri-operative outcomes?: A systematic review and meta-analysis. Eur J Anaesthesiol. 2018;35(7):469-83.

Colantonio L, Claroni C, Fabrizi L, et al. A randomized trial of goal directed vs. standard fluid therapy in cytoreductive surgery with hyperthermic intraperitoneal chemotherapy. J Gastrointest Surg. 2015;19:722-9.

Correa-Gallego C, Tan KS, Arslan-Carlon V, et al. Goal-directed fluid therapy using stroke volume variation for resuscitation after low central venous pressureassisted liver resection: a randomized clinical trial. J Am Coll Surg. 2015:221: 591-601.

Davies SJ, Yates DR, Wilson RJT, et al. A randomised trial of non-invasive cardiac output monitoring to guide haemodynamic optimisation in high risk patients undergoing urgent surgical repair of proximal femoral fractures (ClearNOF trial NCT02382185). Perioper Med (Lond). 2019:8:8.

Donati A, Loggi S, Preiser JC, et al. Goal-directed intraoperative therapy reduces morbidity and length of hospital stay in high-risk surgical patients. Chest. 2007;132:1817-24.

Eappen S, Lane BH, Rosenberg B, et al. Relationship between occurrence of surgical complications and hospital finances. JAMA. 2013;309:1599-606.

Edwards MR, Forbes G, MacDonald N, et al. Optimisation of Perioperative Cardiovascular Management to Improve Surgical Outcome II (OPTIMISE ॥) trial: study protocol for a multicentre international trial of cardiac outputguided fluid therapy with low-dose inotrope infusion compared with usual care in patients undergoing major elective gastrointestinal surgery. BM Open. 2019;9:e023455.

El Sharkawy OA, Refaat EK, Ibraheem AE, et al. Transoesophageal doppler compared to central venous pressure for perioperative hemodynamic monitoring and fluid guidance in liver resection. Saudi J Anaesth. 2013;7:378-86.

Evans D, Doraiswamy V, Prosciak M, Silviera M, et al. Complications associated with pulmonary artery catheters: a comprehensive clinical review. Scand J Surg. 2009;98:199-208.

Funk DJ, HayGlass KT, Koulack J, Harding G, Boyd A, Brinkman R. A randomized controlled trial on the effects of goal-directed therapy on the inflammatory response open abdominal aortic aneurysm repair. Crit Care. 2015;19:247.

Gan TJ, Soppitt A, Maroof M, et al. Goal-directed intraoperative fluid administration reduces length of hospital stay after major surgery. Anesthesiology. 2002;97:820-6.

Gerent ARM, Almeida JP, Fominskiy E, et al. Effect of postoperative goal-directed therapy in cancer patients undergoing high-risk surgery: a randomized clinical trial and meta-analysis. Crit Care. 2018;22:133.

Goepfert MS, Richter HP, Zu Eulenburg C, et al. Individually optimized hemodynamic therapy reduces complications and length of stay in the intensive care unit: a prospective, randomized controlled trial. Anesthesiology. 2013;119:824-36.

Gomez-Izquierdo JC, Trainito A, Mirzakandov D, et al. Goal-directed fluid therapy does not reduce primary postoperative ileus after elective laparoscopic colorectal surgery: a randomized controlled trial. Anesthesiology. 2017;127:36-49.
Grocott MP, Dushianthan A, Hamilton MA, et al. Perioperative increase in global blood flow to explicit defined goals and outcomes following surgery. Cochrane Database Syst Rev. 2012;11:CD004082.

Grocott MPW, Mythen MG, Gan TJ. Perioperative fluid management and clinical outcomes in adults. Anesth Analg. 2005;100:1093-106.

Guyatt GH, Oxman AD, Vist GE, et al. GRADE: an emerging consensus on rating quality of evidence and strength of recommendations. BMJ. 2008; 336:924-6.

Hamilton MA, Cecconi M, Rhodes A. A systematic review and meta-analysis on the use of preemptive hemodynamic intervention to improve postoperative outcomes in moderate and high-risk surgical patients. Anesth Analg. 2011; 112:1392-402.

Han G, Liu K, Xue H, Zhao P. Application of LiDCO-Rapid in peri-operative fluid therapy for aged patients undergoing total hip replacement. Int J Clin Exp Med. 2016:9:4473-8.

Higgins JP, Green S, editor(s). Cochrane Handbook for Systematic Reviews of Interventions Version 5.1.0 (updated March 2011). The Cochrane Collaboration 2011. www.handbook.cochrane.org.

http://www.isrctn.com/ISRCTN14729158.

Jammer I, Tuovila M, Ulvik A. Stroke volume variation to guide fluid therapy: is it suitable for high-risk surgical patients? A terminated randomized controlled trial. Perioper Med (Lond). 2015;4:6.

Jammer I, Ulvik A, Erichsen C, Lodemel O, Ostgaard G. Does central venous oxygen saturation-directed fluid therapy affect postoperative morbidity after colorectal surgery? A randomized assessor-blinded controlled trial. Anesthesiology. 2010;113:1072-80.

Jhanji S, Vivian-Smith A, Lucena-Amaro S, et al. Haemodynamic optimisation improves tissue microvascular flow and oxygenation after major surgery: a randomised controlled trial. Crit Care. 2010;14:R151.

Joosten A, Raj Lawrence S, Colesnicenco A, et al. Personalized Versus Protocolized Fluid Management Using Noninvasive Hemodynamic Monitoring (Clearsight System) in Patients Undergoing Moderate-Risk Abdominal Surgery. Anesth Analg. 2019;129:e8-e12.

Kaufmann KB, Baar W, Rexer J, et al. Evaluation of hemodynamic goal-directed therapy to reduce the incidence of bone cement implantation syndrome in patients undergoing cemented hip arthroplasty - a randomized parallel-arm trial. BMC Anesthesiol. 2018;18:63.

Kaufmann KB, Stein L, Bogatyreva L, et al. Oesophageal Doppler guided goaldirected haemodynamic therapy in thoracic surgery - a single centre randomized parallel-arm trial. Br J Anaesth. 2017;118:852-61.

Kim HJ, Kim EJ, Lee HJ, et al. Effect of goal-directed haemodynamic therapy in free flap reconstruction for head and neck cancer. Acta Anaesthesiologica Scandinavica. 2018;62:903-14.

Kumar L, Rajan S, Baalachandran R. Outcomes associated with stroke volume variation versus central pressure guided fluid replacements during major abdominal surgery. J Anaesthesiol Clin Pharmacol. 2016;32:182-6.

Lobo SM, Salgado PF, Castillo VG, et al. Effects of maximizing oxygen delivery on morbidity and mortality in high-risk surgical patients. Crit Care Med. 2000;28: 3396-404.

Luo J, Xue J, Liu J, Liu B, Liu L, Chen G. Goal-directed fluid restriction during brain surgery: a prospective randomized controlled trial. Ann Intensive Care. 2017; 7:16.

Mayer J, Boldt J, Mengistu AM, Rohm KD, Suttner S. Goal-directed intraoperative therapy based on autocalibrated arterial pressure waveform analysis reduces hospital stay in high-risk surgical patients: a randomized, controlled trial. Crit Care. 2010;14:R18.

McKendry M, McGloin H, Saberi D, Caudwell L, Brady AR, Singer M. Randomised controlled trial assessing the impact of a nurse delivered, flow monitored protocol for optimisation of circulatory status after cardiac surgery. BMJ. 2004:329:258.

McKenny $\mathrm{M}$, Conroy $\mathrm{P}$, Wong $\mathrm{A}$, et al. A randomised prospective trial of intraoperative oesophageal Doppler-guided fluid administration in major gynaecological surgery. Anaesthesia. 2013;68:1224-31.

Mikor A, Trasy D, Nemeth MF, et al. Continuous central venous oxygen saturation assisted intraoperative hemodynamic management during major abdominal surgery: a randomized, controlled trial. BMC Anesthesiol. 2015;15:82.

Moppett IK, Rowlands M, Mannings A, Moran CG, Wiles MD, Investigators N. LiDCO-based fluid management in patients undergoing hip fracture surgery under spinal anaesthesia: a randomized trial and systematic review. $\mathrm{Br}$ J Anaesth. 2015;114:444-59. 
Mythen MG, Webb AR. Perioperative plasma volume expansion reduces the incidence of gut mucosal hypoperfusion during cardiac surgery. Arch Surg. 1995;130:423-9.

Noblett SE, Snowden CP, Shenton BK, Horgan AF. Randomized clinical trial assessing the effect of Doppler-optimized fluid management on outcome after elective colorectal resection. Br J Surg. 2006;93:1069-76.

Osawa EA, Rhodes A, Landoni G, et al. Effect of perioperative goal-directed hemodynamic resuscitation therapy on outcomes following cardiac surgery: a randomized clinical trial and systematic review. Crit Care Med. 2016;44: 724-33.

Pearse R, Dawson D, Fawcett J, Rhodes A, Grounds RM, Bennett ED. Early goaldirected therapy after major surgery reduces complications and duration of hospital stay. A randomised, controlled trial [ISRCTN38797445]. Crit Care. 2005;9:R687-93.

Pearse RM, Harrison DA, MacDonald N, et al. Effect of a perioperative, cardiac output-guided hemodynamic therapy algorithm on outcomes following major gastrointestinal surgery: a randomized clinical trial and systematic review. JAMA. 2014;311:2181-90.

Pearse RM, Moreno RP, Bauer P, et al. Mortality after surgery in Europe: a 7 day cohort study. Lancet. 2012;380:1059-65.

Peng K, Li J, Cheng H, Ji FH. Goal-directed fluid therapy based on stroke volume variations improves fluid management and gastrointestinal perfusion in patients undergoing major orthopedic surgery. Med Princ Pract. 2014;23: 413-20.

Pestana D, Espinosa E, Eden A, et al. Perioperative goal-directed hemodynamic optimization using noninvasive cardiac output monitoring in major abdominal surgery: a prospective, randomized, multicenter, pragmatic trial: POEMAS Study (PeriOperative goal-directed therapy in Major Abdominal Surgery). Anesth Analg. 2014;119:579-87.

Phan TD, D'Souza B, Rattray MJ, Johnston MJ, Cowie BS. A randomised controlled trial of fluid restriction compared to oesophageal Doppler-guided goal-directed fluid therapy in elective major colorectal surgery within an Enhanced Recovery After Surgery program. Anaesth Intensive Care. 2014;42:752-60.

Polonen P, Ruokonen E, Hippelainen, et al. A prospective, randomized study of goal-directed hemodynamic therapy in cardiac surgical patients. Anesth Analg. 2000;90:1052-9.

Reisinger KW, Willigers HM, Jansen J, et al. Doppler-guided goal-directed fluid therapy does not affect intestinal cell damage but increases global gastrointestinal perfusion in colorectal surgery: a randomized controlled trial. Colorectal Dis. 2017;19:1081-91.

Ruscic KJ, Grabitz SD, Rudolph MI, Eikermann M. Prevention of respiratory complications of the surgical patient: actionable plan for continued process improvement. Curr Opin Anaesthesiol. 2017;30:399-408.

Salzwedel C, Puig J, Carstens A, et al. Perioperative goal-directed hemodynamic therapy based on radial arterial pulse pressure variation and continuous cardiac index trending reduces postoperative complications after major abdominal surgery: a multi-center, prospective, randomized study. Crit Care. 2013;17:R191.

Sandham JD, Hull RD, Brant RF, et al. A randomized, controlled trial of the use of pulmonary-artery catheters in high-risk surgical patients. N Engl J Med. 2003; 348:5-14.

Scheeren TW, Wiesenack C, Gerlach H, Marx G. Goal-directed intraoperative fluid therapy guided by stroke volume and its variation in high-risk surgical patients: a prospective randomized multicentre study. J Clin Monit Comput. 2013:27:225-33.

Schmid S, Kapfer B, Heim M, et al. Algorithm-guided goal-directed haemodynamic therapy does not improve renal function after major abdominal surgery compared to good standard clinical care: a prospective randomised trial. Crit Care. 2016;20:50.

Senagore AJ, Emery T, Luchtefeld M, Kim D, Dujovny N, Hoedema R. Fluid management for laparoscopic colectomy: a prospective, randomized assessment of goal-directed administration of balanced salt solution or hetastarch coupled with an enhanced recovery program. Dis Colon Rectum. 2009;52:1935-40.

Shoemaker WC, Appel PL, Kram HB, Waxman K, Lee TS. Prospective trial of supranormal values of survivors as therapeutic goals in high-risk surgical patients. Chest. 1988;94:1176-86.

Sinclair S, James S, Singer M. Intraoperative intravascular volume optimisation and length of hospital stay after repair of proximal femoral fracture: randomised controlled trial. BMJ. 1997:315:909-12.
Srinivasa S, Taylor MH, Singh PP, YU TC, Soop M, Hill AG. Randomized clinical trial of goal-directed fluid therapy within an enhanced recovery protocol for elective colectomy. Br J Surg. 2013;100:66-74.

Stens J, Hering JP, van der Hoeven CWP, et al. The added value of cardiac index and pulse pressure variation monitoring to mean arterial pressureguided volume therapy in moderate-risk abdominal surgery (COGUIDE): a pragmatic multicentre randomised controlled trial. Anaesthesia. 2017;72: 1078-87.

Ueno S, Tanabe G, Yamada H, et al. Response of patients with cirrhosis who have undergone partial hepatectomy to treatment aimed at achieving supranormal oxygen delivery and consumption. Surgery. 1998;123:278-86.

Valentine RJ, Duke ML, Inman MH, et al. Effectiveness of pulmonary artery catheters in aortic surgery: a randomized trial. J Vasc Surg. 1998;27:203-11.

Van der Linden PJ, Dierick A, Wilmin S, Bellens B, De Hert SG. A randomized controlled trial comparing an intraoperative goal-directed strategy with routine clinical practice in patients undergoing peripheral arterial surgery. Eur J Anaesthesiol. 2010:27:788-93.

Venn R, Steele A, Richardson P, Poloniecki J, Grounds M, Newman P. Randomized controlled trial to investigate influence of the fluid challenge on duration of hospital stay and perioperative morbidity in patients with hip fractures. $\mathrm{Br}$ J Anaesth. 2002;88:65-71.

Wakeling HG, McFall MR, Jenkins CS, et al. Intraoperative oesophageal Doppler guided fluid management shortens postoperative hospital stay after major bowel surgery. Br J Anaesth. 2005;95:634-42.

Weinberg L, lanno D, Churilov L, et al. Restrictive intraoperative fluid optimisation algorithm improves outcomes in patients undergoing pancreaticoduodenectomy: A prospective multicentre randomized controlled trial. PLoS One. 2017;12:e0183313.

Weinberg L, lanno D, Churilov L, et al. Goal directed fluid therapy for major liver resection: A multicentre randomized controlled trial. Ann Med Surg (Lond). 2019:45:45-53.

Wilson J, Woods I, Fawcett J, et al. Reducing the risk of major elective surgery: randomised controlled trial of preoperative optimisation of oxygen delivery. BMJ. 1999;318:1099-103.

Wu J, Ma Y, Wang T, Xu G, Fan L, Zhang Y. Goal-directed fluid management based on the auto-calibrated arterial pressure-derived stroke volume variation in patients undergoing supratentorial neoplasms surgery. Int J Clin Exp Med. 2017;10:3106-14.

Xu H, Shu SH, Wang D, Chai XQ, Xie YH, Zhou WD. Goal-directed fluid restriction using stroke volume variation and cardiac index during one-lung ventilation: a randomized controlled trial. J Thorac Dis. 2017:9:2992-3004.

Yin K, Ding J, Wu Y, Peng M. Goal-directed fluid therapy based on noninvasive cardiac output monitor reduces postoperative complications in elderly patients after gastrointestinal surgery: A randomized controlled trial. Pak J Med Sci. 2018;34:1320-5.

Zakhaleva J, Tam J, Denoya PI, Bishawi M, Bergamaschi R. The impact of intravenous fluid administration on complication rates in bowel surgery within an enhanced recovery protocol: a randomized controlled trial. Colorectal Dis. 2013;15:892-9.

Zeng K, Li Y, Liang M, Gao Y, Cai H, Lin C. The influence of goal-directed fluid therapy on the prognosis of elderly patients with hypertension and gastric cancer surgery. Drug Des Devel Ther. 2014;8:2113-9.

Zhang J, Chen CQ, Lei XZ, Feng ZY, Zhu SM. Goal-directed fluid optimization based on stroke volume variation and cardiac index during one-lung ventilation in patients undergoing thoracoscopy lobectomy operations: a pilot study. Clinics (Sao Paulo) 2013; 68: 1065-1070.

\section{Publisher's Note}

Springer Nature remains neutral with regard to jurisdictional claims in published maps and institutional affiliations. 